\title{
The Design of Drill Rod Interfaces and Their Auxiliary Mechanism DRIAM
}

\author{
Julien Marcil*, Patrick Sabbag*, Jean-Philippe Nolet*, \\ Howard Jones ${ }^{* *}$, and Alexei Morozov* \\ *Department of Mechanical Engineering, McGill University, Montreal, Quebec, Canada \\ ${ }^{* *}$ MD Robotics, Brampton, Ontario, Canada \\ julmar@cim.mcgill.ca_alexvit@cim.mcgill.ca
}

\begin{abstract}
In the paper the design of the drill rod interfaces and their auxiliary mechanism (DRIAM) for space exploration systems is described. The DRIAM project is sub divided into 3 parts: the drill rod segment, the auxiliary mechanism, and the drill rod storage mechanism. This system is to be integrated into a Mars land rover. The short drill rod segments have locking mechanisms inside allowing them to lock to each other, thus forming a two-meter drill string. These rods are stored in a storage mechanism. The auxiliary mechanism translates segments between the storage mechanism and the drill string. The system has a total of five degrees-of-freedom: four active, where the auxiliary mechanism has three dof, and the storage mechanism has one, and one passive located in the drill rod segment activated by the auxiliary mechanism.
\end{abstract}

\section{Introduction}

There is an evidence that liquid water once covered the Martian surface, as it is also known that the red planet was once enveloped by a nitrogen-rich atmosphere. This leads to the idea that Mars could have sustained life, and perhaps still does today below its surface. One of the next crucial steps in the exploration of Mars is the analysis of the Martian soil, located several meters deep, for signs of favorable conditions for the evolution of life [1]. To accomplish this feat, a drilling and sample acquisition mechanism that can be integrated into a Mars land rover must be developed.

MD Robotics has developed drill concepts for the 2009 Mars SmartLander program in conjunction with NASA and the CSA and for the ExoMars and Mars Sample Return missions of the European Space Agency. As a next step MD Robotics has set up the task of designing and building prototypes of critical aspects of the drill to prove the concepts [2]. The project was divided in two: the sample acquisition mechanism, and the drill rod segments and their auxiliary mechanism. We were responsible for the second part of the project, the DRIAM.

Drill rod segments that are assembled to form long drill strings are found in mining and many different arrangements, allowing rods to lock to each other [3]. MD Robotics has worked with partners Norcat and EVC, both involved in mining, to develop such a device [4]. Mechanisms that can manipulate such drill rod segments are abundantly found in Computer Numerical Control (CNC) machines but are generally pneumatic, which is not desirable for a mission to Mars [5]. Furthermore, they are both generally not restrained by size requirements. The difficulty of this project arises in the extremely small volume allocated to such devices.

As it is impractical to carry a drill rod exceeding a couple decimeters in length in the Mars land rover, a system composed of several short drill rod segments that can be attached and detached remotely must be designed.

These rods must be stored into a compact package, since they will be located on the rover. The rods must also incorporate an interface allowing them to lock and unlock from each other, transmit eight electrical signals, and transmit the necessary torque and thrust entailed in such a drilling operation.

In conjunction with MD Robotics, we have designed and built a prototype of the drill rod segments, the auxiliary mechanism that manipulates the rods, as well as the rod storage mechanism. Our design does not include the mechanism responsible for drilling. 
The objectives of this project were to develop and test a robust system capable of assembling a 2-meter long drill string, and storing its segments compactly.

\section{Problem Definition}

With our client from MD Robotics, the requirements for the project were defined. The system was divided into three main sub-components: the rod segments, the auxiliary mechanism, which manipulates the rod segments, and the drill rod storage mechanism. It was initially unclear whether the auxiliary mechanism and the storage mechanism would consist of two separate mechanisms; some preliminary concepts had the two incorporated into a single mechanism. The definition of the problem, and its possible solutions, evolved with the project.

The entire system must have a maximum height of 40 $\mathrm{cm}$, length of $45 \mathrm{~cm}$, and width of $40 \mathrm{~cm}$, to fit into the envelope allocated to it within the rover. The system must contain the means of actuation and sensing. Total power consumption must not exceed an average of $10 \mathrm{~W}$ per cycle, excluding the drilling itself, and a peak of $20 \mathrm{~W}$ is allowed for a maximum duration of 20 seconds. The entire device must be activated and controlled autonomously. Design must be done with space-rated material; however, standard commercially available materials can be used for the construction of the prototype.

The outer diameter of the rod segments cannot exceed $20 \mathrm{~mm}$ excluding the helicoids used to remove the soil. The length of the drill string must be of $2 \mathrm{~m}$. The rod segments and their respective interfaces must transmit $2.5 \mathrm{Nm}$ of torque and $500 \mathrm{~N}$ of thrust. The drill rod interfaces must allow remote attachment and detachment. The drill rod interfaces must transmit eight electrical signals.

The auxiliary mechanism must acquire, move, attach, and detach the individual rod segments. And finally, the storage mechanism must constrain the movement of the drill rods when in storage.

\section{Design process}

When approaching a project of this magnitude, it is important to break it down into sub-problems, which can be surmounted in a sequential manner. However, the design of this system (Fig.1) does not allow the three sub-components to be developed independently and joined when finished. Thus, they must be developed in parallel.
The first issue to address was whether the rods would lock together with a purely linear motion or with a rotary motion. This characteristic dictates the design of the drill rod interfaces and the motion required from the auxiliary mechanism. Furthermore, the auxiliary mechanism motion dictates the motion required from the storage mechanism, as the auxiliary mechanism must acquire the rod segments from the storage.

Devices using two threaded surfaces to lock together are the most frequently seen of the two. These devices require a rotary motion for locking. Such a design could be implemented on the rod segments [6]. The joining parts would be screwed together with a certain preload. In our particular application the preloaded would have to be higher than $2.5 \mathrm{Nm}$, so that when the drill string is run in reverse to bring it up, and an external load of $2.5 \mathrm{Nm}$ is present, the segments do not unscrew and detach from each other.

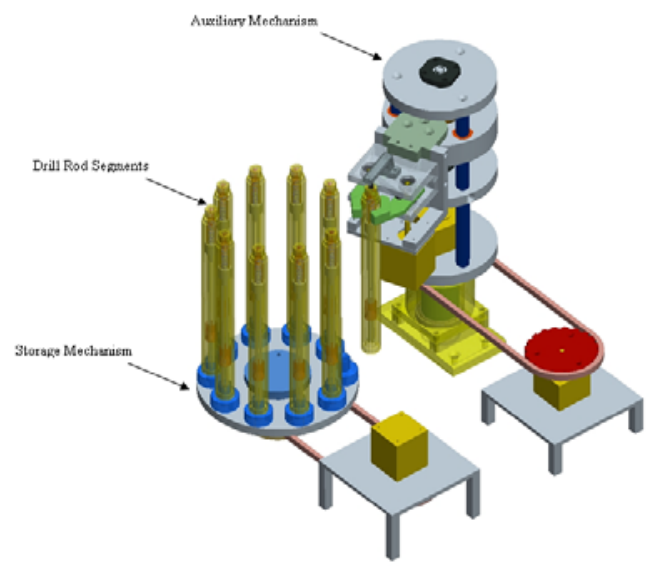

(a)

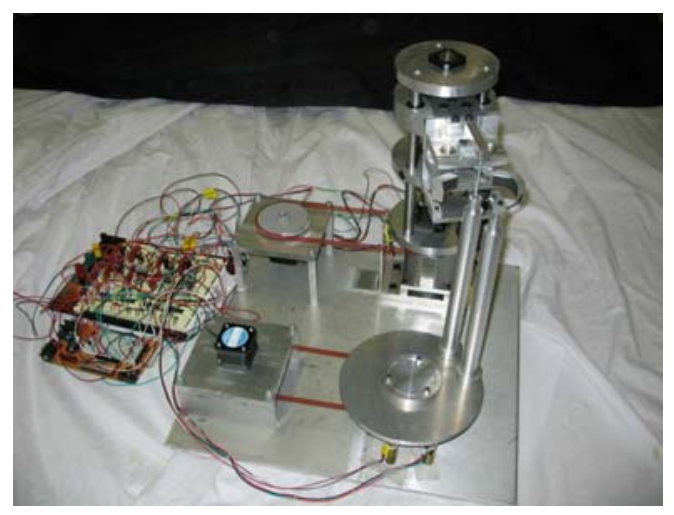

(b)

Fig.1. DRIAM project (a) model; (b) prototype

Locking mechanisms that require pure linear motion are also common. In our case however, such a device 
is restrained to operate in an area with a diameter smaller than $20 \mathrm{~mm}$.

The two options were studied and it was found that a linear locking mechanism in the rods would render a simpler auxiliary mechanism. MD Robotics also showed greater interest in the development of a linearly locking drill rod segment, as they were more familiar with rotary locking drill rod segments and wanted linear locking drill rod segment concepts to be explored. With such a design, the auxiliary mechanism would only need to move the rods vertically and horizontally, eliminating the third degree-of-freedom.

With the knowledge of the motion required for the locking of the drill rod segments, it was possible to proceed with the design. The design of the drill rod segments is first examined, followed with that of the auxiliary mechanism and finally that of the storage mechanism.

\section{DRIAM Architecture}

\subsection{Rod Segments}

A drill string measuring two meters in length, capable of transmitting eight electrical signals, is required to sample the Martian soil. To accomplish this, it was decided that 10 drill rod segments (Fig.2) measuring $20 \mathrm{~mm}$ each will be assembled to form the two meter long drill string.

The body of each $20 \mathrm{~mm}$ rod must be able to withstand $500 \mathrm{~N}$ of thrust and $2.5 \mathrm{Nm}$ of torque. The axial load is applied in two instances; when drilling down into the Martian surface, and when drilling out of the Martian earth. Therefore the $500 \mathrm{~N}$ is transmitted through the rod body when drilled down, and through the locking mechanism when pulling the rod string out of the Martian surface. Two simple stress analyses were done to ensure that these requirements were satisfied. The torque transmission is discussed later as it is done by the locking mechanism.

The mechanism inside the rods (Fig.3) responsible for the locking and unlocking is similar to a collet as seen in Fig.4 and will therefore be called a collet in this project. The collet is collapsed when an axial load is applied to the unlocking mechanism releasing the rod segment from the drill string or from the storage mechanism since the rods attach to each other in the same manner as they attach to the storage mechanism (Fig.5). When the force is removed, a spring pushes the slider back up, allowing the collet to expand. It was therefore essential to design the auxiliary mechanism in conjunction with the rod segments as it would activate the locking and unlocking mechanism as can be seen in section 4.2.1.

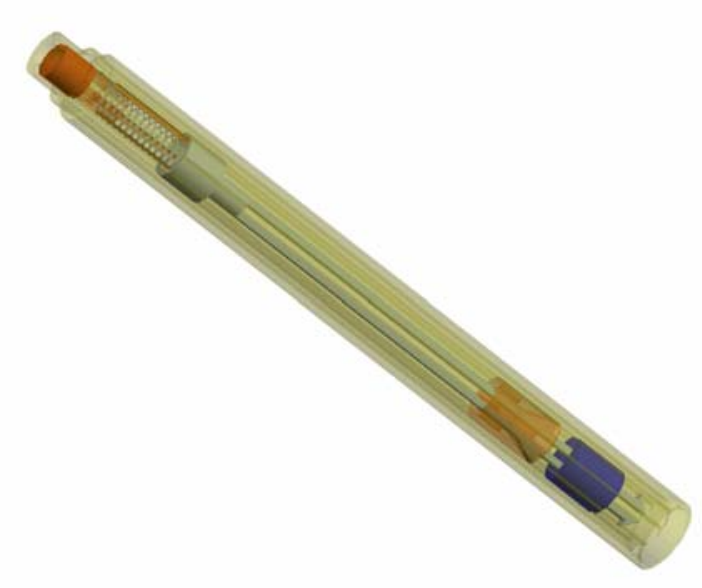

Fig.2. Rod segment model

To calculate the necessary force required to collapse the collet, the locking mechanism is modeled as a combination of two cantilevered beams, as seen in Fig.6.

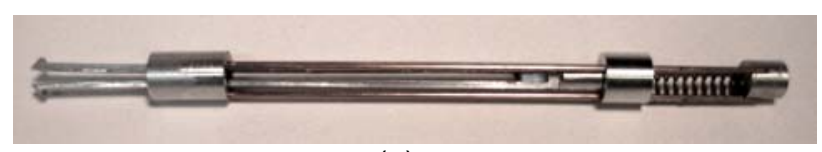

(a)

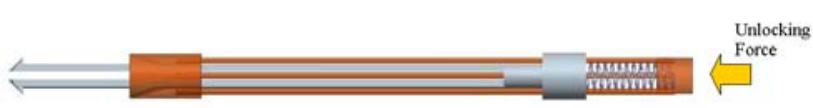

(b)

Fig.3. Rod locking mechanism (a) prototype; (b) model

The drill rods will need the capability to transmit control signals. An electrical interface, transmitting the signals down to the acquisition mechanism at the end of the drill string, requires precise radial positioning. The rod segments must therefore have the ability to self-index since electrical contacts on the drill rod interfaces need to align when rods are connected. A rod must position itself into proper orientation without rotational motion from the auxiliary mechanism since it was established that the rods would lock together without such motion to keep the auxiliary mechanism simple. This is achieved with the mechanism depicted in Fig.7. 


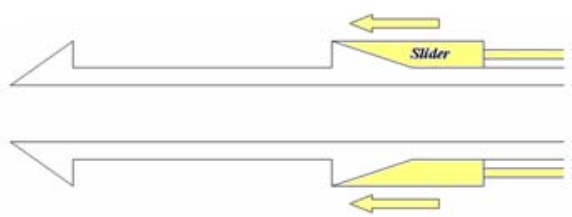

Fig.4. Rod slider collapsing collet
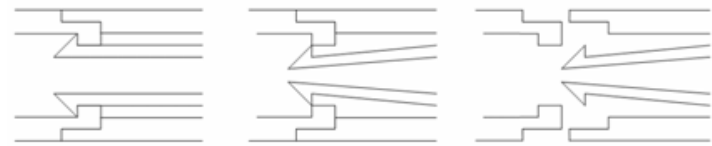

Fig.5. Rod unlocking when collet collapsed

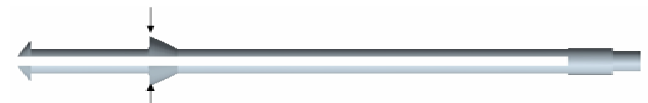

(a)

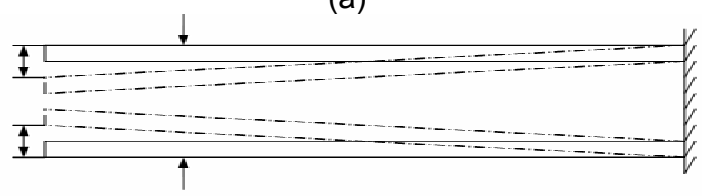

(b)

Fig.6. (a) Collet; (b) collet modeled as cantilevered beam

As can be seen, the collet collapses when the rod is inserted into another drill segment no matter its radial orientation. The rods are partly locked when initially inserted; their movement in the axial direction is restricted, but they are still free to rotate. This is due to the fact that the collet partially expands. The collet only fully expands and therefore locks into radial position when rotated, aligning the rod segments into there desired orientation which aligns the electrical contacts.

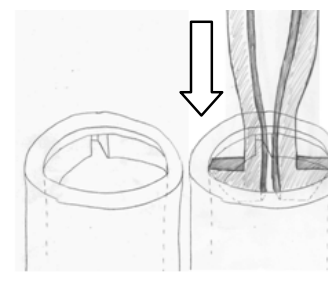

(a)

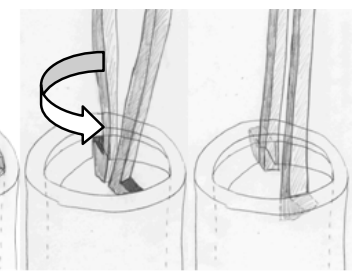

(c) (d)
Fig.7. Rod segment self-indexing:

(a) rod; (b) collet inserted; (c) in position;

(d) expanded and locked

The rotation of the rod would be exerted by the same mechanism responsible for the drilling. A controller monitoring the applied torque will see a sharp increase when the collet expands into position fully locking the rods together signaling that the electrical contacts are aligned. At this point, the newly connected segment would be ready for drilling (Fig.8).

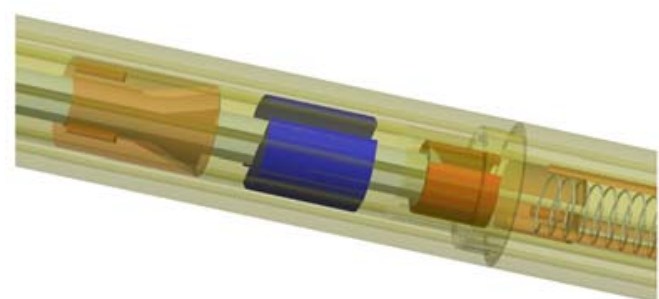

Fig.8. Rod locked to other rod close-up view

The collet, when expanded into position (Fig.7d), transmits the torque from rod segment to rod segment. The collet therefore serves two purposes, the selfindexing locking of rods and the transmission of torque. The design element that ensures that the torque can be transmitted without failure is represented in blue in Fig.9.

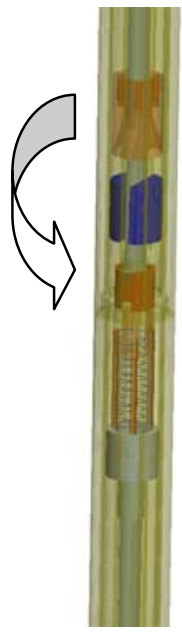

Fig.9. Torque transmitting part of rod

If this element was not present the rod locking mechanism alone, as seen in Fig.3, would be loaded when transmitting the torque. Since the collet was designed to be easily collapsed, it has a considerable length measuring $150 \mathrm{~mm}$. This length greatly lowers the amount of torque it can sustain without failing. It was therefore necessary to introduce a feature to the rod that would transfer the load to the body of the rod. 
The rods can lock together and align themselves into a given orientation; they can therefore transmit an electrical signal through isolated conducting surfaces. This is the concept used in this design. When the rods are aligned, the holes carrying the wires are therefore also aligned. At each end of the rod the wire is isolated from the rod's conductive body as seen in Fig. 10.

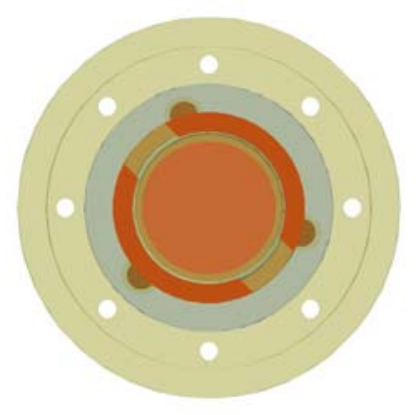

(a) Top view of rod with visible wires
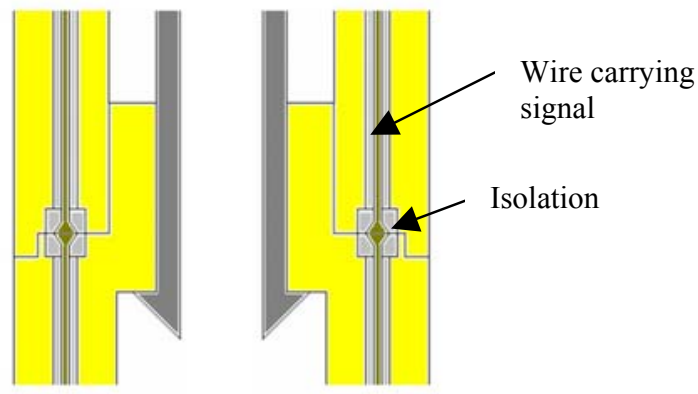

(c) Electrical interface representation

Fig.10. Electrical interface

\subsection{Auxiliary Mechanism:}

The auxiliary mechanism is a three degree-of-freedom manipulator. The hand, which grips the rods, can move vertically and horizontally around its axis as seen in Fig.11. Its function is to unlock the rods from either the storage mechanism or from each other, and to bring them to their desired location, either back to the storage mechanism or to the deployed drill string. The auxiliary mechanism includes three subcomponents: the auxiliary tower, the hand, and the base.

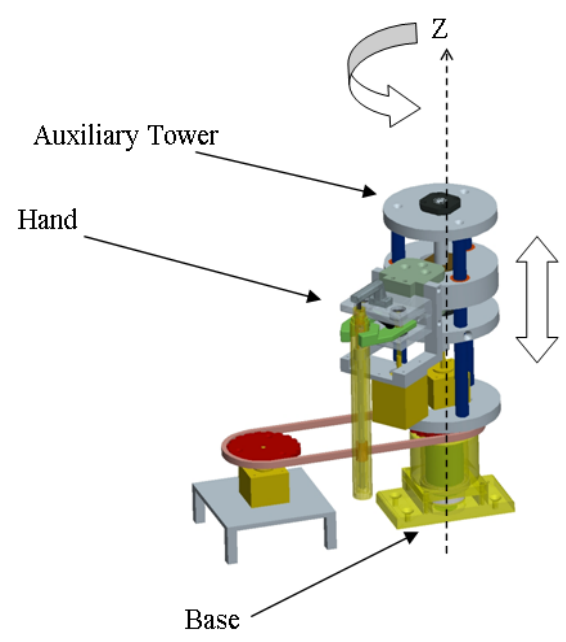

Fig.11. Auxiliary mechanism model

\subsubsection{Auxiliary Mechanism Hand}

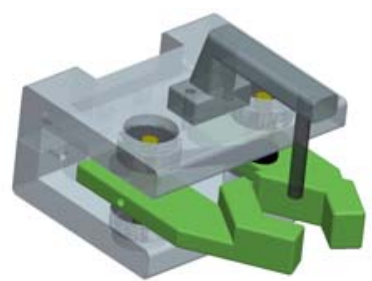

Fig.12. Auxiliary mechanism hand

The hand of the auxiliary mechanism (Fig.12) is responsible for the acquisition of the drill rod segments; it must unlock the rod segments and securely grip them.

As discussed in section 4.1, a vertical force is required to unlock the drill rod segment from the drill rod string or the storage mechanism. This force is applied by the pin attached to the hand assembly as seen in Fig.13. The pin pushes down on the unlocking mechanism, sliding the slider down the collet, collapsing it, and consequently unlocking the rod.
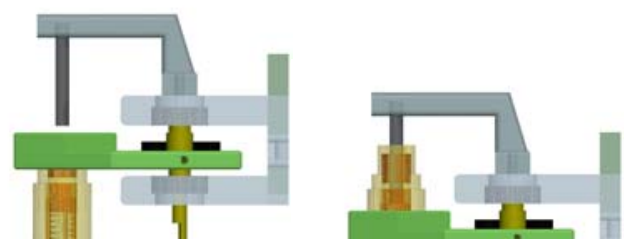

Fig.13. Hand pin unlocking rod segment

The rod remains in an unlocked configuration until it is released from the hand. 


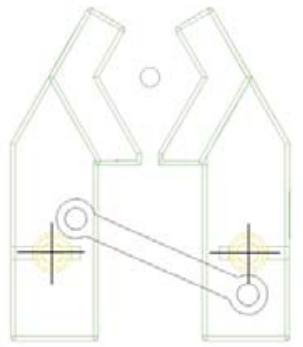

(a)

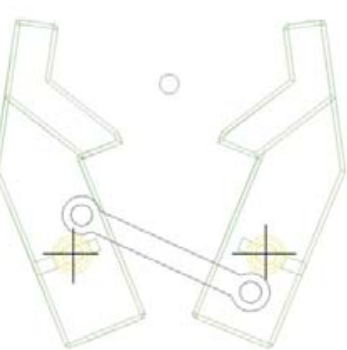

(b)
Fig.14. Four-bar mechanism in (a) close hand position and (b) open hand position

The hand grips the rod in a fashion similar to that in which a human would do so with his fingers. The desired motion for the two fingers of the auxiliary mechanism's hand is achieved with a four-bar mechanism (Fig.14).

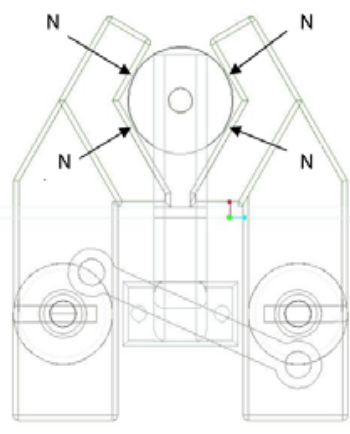

(a)

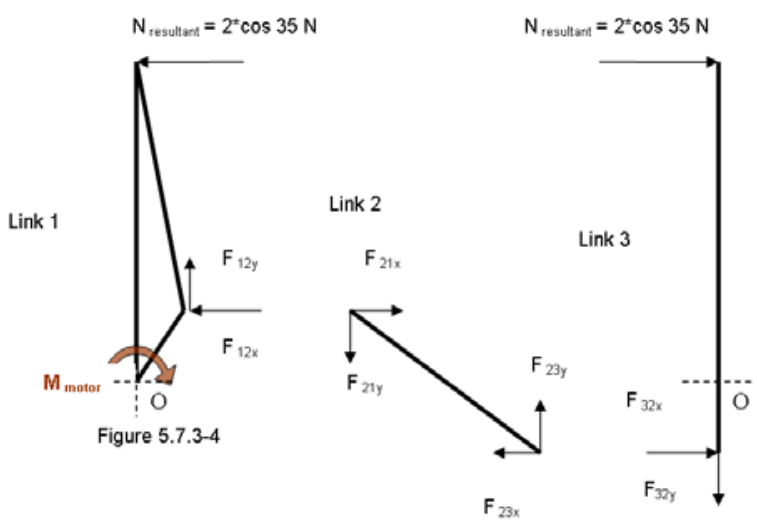

(b)

Fig.15. (a) Force applied to rod through 4-bar linkage (b) Free body diagram of 4-bar linkage

The two fingers are driven by a single motor located directly below the first finger's axis of rotation. The force is transmitted to the second finger via the link coupling them (Fig.15). The torque requirement for the motor was found assuming the friction factor between the rod and the finger was 0.9 , corresponding to the friction factor between aluminum and rubber. Rubber will be placed on the areas of contact of the fingers.

\subsubsection{Auxiliary Mechanism Base}

The auxiliary mechanism base (Fig.16) supports the weight of the auxiliary mechanism and the load produced when a rod is picked up. The design of the base, which is composed of two radial bearings and one thrust bearing, was done to withstand a $88.3 \mathrm{~N}$ axial load and a $94.0 \mathrm{~N}$ radial load which were found with a simple analysis.
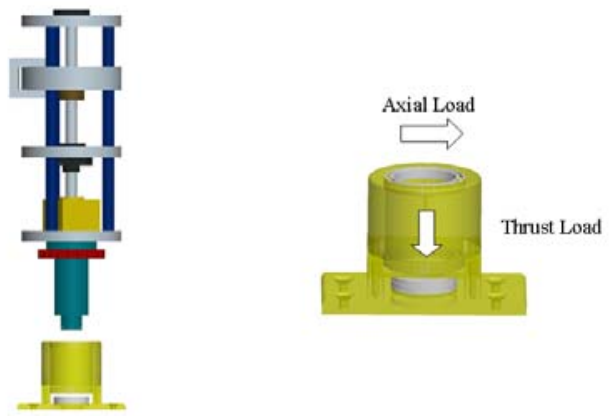

Fig.16. Auxiliary mechanism base

\subsubsection{Auxiliary Mechanism Tower}

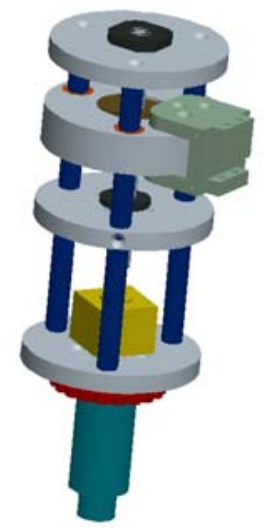

Fig.17. Auxiliary mechanism tower

The auxiliary mechanism tower (Fig.17) was designed to provide the necessary vertical and horizontal motion of the hand. A motorized ball screw assembly 
lifts and lowers the hand while a motorized sprocket and belt assembly rotates the entire tower.

The mass to be vertically lifted and lowered is roughly $5 \mathrm{~kg}$. The mechanical advantage of a ball screw is therefore used to move this mass (Fig.18). The shoulder, the component directly attached to the ball screw nut, slides along three guides. To minimize friction, linear ball bearings are used between the shoulder and the hardened steel guides.

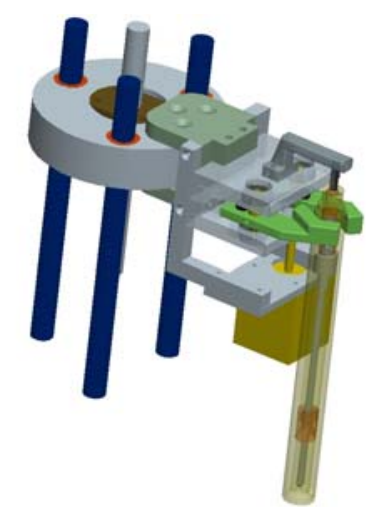

Fig.18. Ball screw and guide

\subsection{Storage Mechanism}

The storage mechanism must simply bring the drill rod segments to a predestine position in which they are to be picked up by the auxiliary mechanism. The storage mechanism must move in increments of $36^{\circ}$ as there are 10 equally placed drill rod segments locked to the storage mechanism. This is easily accomplished with a sprocket and belt assembly driven by a stepper motor with a $1.8^{0}$ step angle as seen in Fig.19. Twenty steps will move the storage plate by exactly $36^{0}$ since a one to one gear ratio is used.

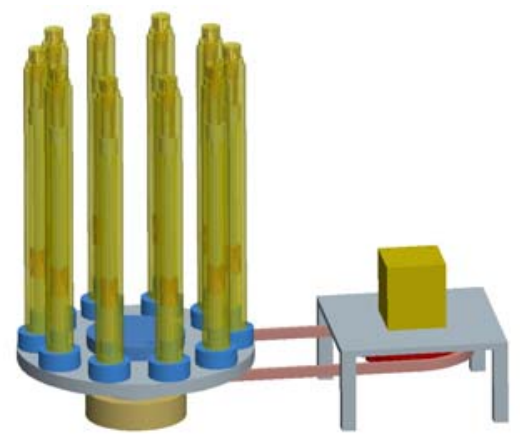

Fig.19. Storage mechanism model

The important feature of the storage mechanism is the clip on which the rods lock themselves (Fig.20). This clip is almost identical to the top of a drill rod segment; the rods therefore lock to the storage mechanism in the same way that the rods lock to each other. The difference is that there are no slits in the clip as there are in the top of a rod segment that allow the collet to fully expand in only one orientation. The rod can therefore lock itself to the clip in any orientation, but will be free to rotate.

\subsection{DRIAM Layout}

The layout of the sub-components of our project will enable them to work together as intended.

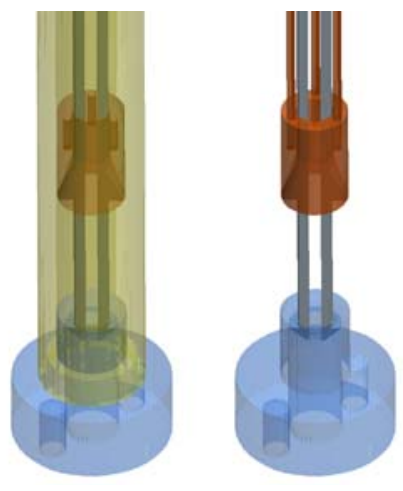

Fig.20. Rod locked to storage clip

The most important aspect of the layout is the distance between the axis of rotation of the auxiliary mechanism and that of the storage magazine's plate. The arm of the auxiliary mechanism, the rod to be picked up, and the axis of rotation of the storage mechanism do not form a $180^{\circ}$ angle when the hand is in position to grip a rod. If the layout was in this manner, the rod being gripped by the hand would hit the next rod waiting to be moved into position to be picked up as seen in Fig.21.

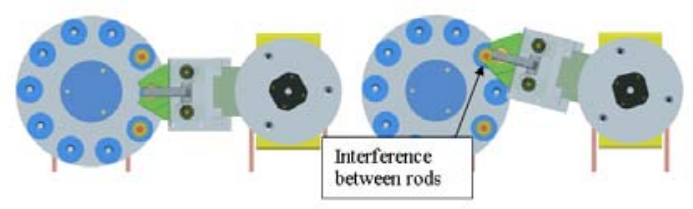

Fig.21. Improper layout causing interference

Instead, they are arranged with a $165^{\circ}$ angle between them as seen in Fig.22. This allows room for the rod to pass with no interference.

Once the positions in which the auxiliary mechanism and the storage mechanism must be in for the pick up of a rod are known, they can easily be implemented through the programming of the controller. 

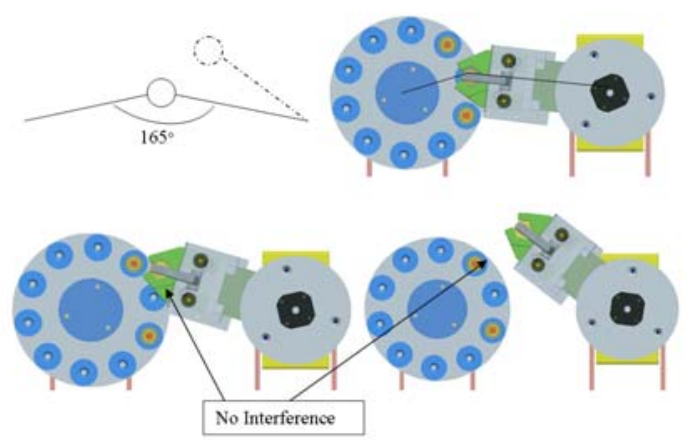

Fig.22. Proper DRIAM layout, no interference

\section{Controller Architecture}

The DRIAM system has four active degrees of freedom, each controlled by a stepper motor. The commands that control the stepper motors are generated by a microcontroller (basic stamp II), sent to motor drivers, then sent to motor driver amplifiers, and finally received by the stepper motors. This particular configuration was used for the prototype (Fig.23).

\subsection{Stepper Motor}

Stepper motors' characteristics make them ideal for this project's particular applications. They can be used feedfoward, no feedback required (encoders, potentiometers, etc). Our application only requires the control of position, the control of the velocity is not essential for the proper functioning of our system. Hall Effect sensors will however be used as limiting switches to ensure that the desired positions are achieved. They will in fact determine the home position, from which the system will travel keeping track of its displacements.

\subsection{Microcontroller}

A basic stamp bs2-p40 microcontroller is used to control our system. Its 32 input/output pins are ideal for our application since we are driving four stepper motors each using two limiting switches. The Hall Effect sensors, which detect a magnetic field, will let our microcontroller know when a given position is reached.

The microcontroller will send three signals to each stepper motor: enable/disable, a clock, and a direction. It will also be receiving two signals for each motor sent by the limiting switches.

\subsection{Motor Drivers}

The motor drivers take the commands from the microcontroller and generate the four phase signals necessary to drive the stepper motors. The drivers translate a rising edge of the clock signal into a step.

The next stage is amplification. The subsequent drivers act as amplifier by generating four powered signals before they reach the motors. They are in fact dual h-bridges.

The motors are supplied by 5 volts and draw 1 amp; the system is therefore never exceeding the 10 Watt limit since only one motor turns at any given time drawing 5 Watts.

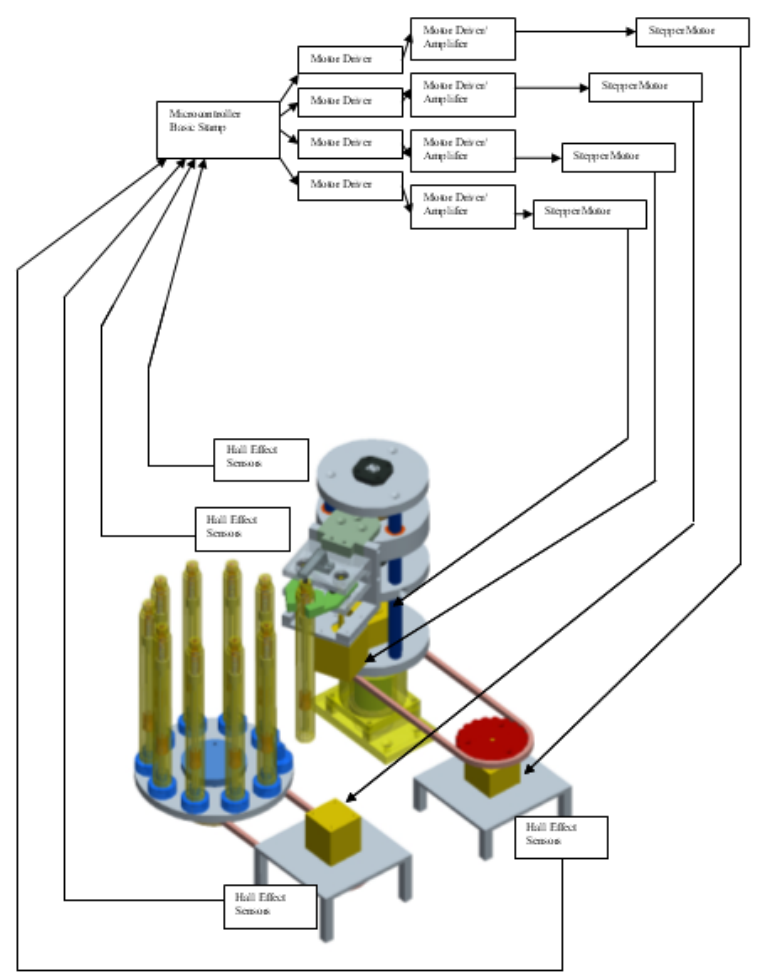

Fig.23. Control information diagram

\section{Conclusions}

The initial decision that was taken to have a linearly activated locking and unlocking mechanism transferred the complexity from the auxiliary mechanism to the rod segment's locking mechanism. A rotationally engaged locking mechanism would have brought more complexity to the auxiliary 
mechanism, however potentially greatly reducing that of the drill rod locking mechanism. This could be advantages since there is considerably less volume constraints imposed on the auxiliary mechanism.

The drill rods of the DRIAM project are the most complex elements of the system. They consist of a rod inside of which a spring and collet assembly is used to lock onto an adjacent rod or the storage plate. The rods are complex because of their size limitation. It was found that they can easily sustain a load of $500 \mathrm{~N}$ and a torque of $2.5 \mathrm{Nm}$. Therefore, a recommendation would be to increase the inner diameter of the rods in order to increase the interior volume, allocating more room for the locking and unlocking mechanism.

The auxiliary mechanism's difficulties arise from the potential alignment problems encountered with such designs. The auxiliary mechanism's shoulder should also be designed in such a way as to reduce the weight of the auxiliary mechanism.

The storage mechanism consists of a simple design allowing for easy manufacturing and high reliability. Its capacity to hold the rods while on its voyage from the Earth to the red planet however must be examined.

The next design iteration should have a considerable focus on optimizing the system's total weight since it is to be sent to Mars on a rover.

\section{Acknowledgements}

The authors would like to thank professor Jorge Angeles who helped us on key issues such as the hand design, while the team at McGill's Mechanical Engineering Machine Shop helped us tremendously with the machining ensuring that we would have a working prototype for the presentation

\section{References}

[1] Bergeen Laurence (2000), Voyage to Mars: NASA's Search for Life Beyond Earth, RiverHead Books, New York.

[2] http://www.mdrobotics.ca/wwdframe.html

[3] Australian Drilling Industry Training Committee Limited (1997), Drilling: the manual of methods, applications, and management, 4th edition, Boca Raton, Fla., CRC Press.
[4] http://www.norcat.org/

[5] Crandell Thomas M. (2003), CNC Machining and Programming: An Introduction, Second edition, New York, Industrial Press.

[6] C. P. Chugh (1992), High Technology in Drilling and Exploration, Rotterdam, A.A. Balkema 